\title{
2016 European guideline on the management of non- gonococcal urethritis published
}

\author{
H Moi ${ }^{12}$, PJ Horner 34 \\ 1. Olafia Clinic, Oslo University Hospital, Oslo, Norway \\ 2. Institute of Medicine, University of Oslo, Oslo, Norway \\ 3. School of Social and Community Medicine, University of Bristol, Bristol, United Kingdom \\ 4. Bristol Sexual Health Centre, University Hospitals Bristol NHS Foundation Trust, Bristol, United Kingdom
}

Correspondence: Harald Moi (harald.moi@medisin.uio.no)

Citation style for this article:

Moi H, Horner PJ. 2016 European guideline on the management of non-gonococcal urethritis published. Euro Surveill. 2016;21(22):pii=30248. DOI: http://dx.doi. org/10.2807/1560-7917.ES.2016.21.22.30248

Article published on 02 June 2016

On 4 May 2016, the evidence-based '2016 European Guideline on the management of non-gonococcal urethritis' was published online [1]. This guideline is a comprehensive updated version of the European guideline from 2009 [2] and provides up-to-date and detailed guidance regarding aetiology, clinical features, diagnosis, testing, treatment and general management of symptomatic non-gonococcal urethritis in Europe.

The most common organisms implicated are Chlamydia trachomatis and Mycoplasma genitalium, with the latter perhaps causing more symptoms [3]. Testing male patients with urethritis for $M$. genitalium, preferably with screening for macrolide resistance, is highly likely to improve clinical outcomes [4]. Testing symptomatic patients for M. genitalium is therefore recommended.

Of major concern is the increasing azithromycin resistance of $M$. genitalium [5]. Azithromycin, especially single dosage of $1 \mathrm{~g}$, is associated with the development of macrolide resistance in $M$. genitalium, and is likely to increase the circulation of macrolide-resistant strains in the population [5]. Consequently, single dose azithromycin is no longer recommended as first-line treatment for non-gonococcal urethritis.

Updates to the guideline include recommendations on the diagnosis, testing and treatment of non-gonococcal urethritis:

Urethritis should be confirmed by urethral smear microscopy in symptomatic patients.

- Symptoms and negative urethral smear: No empirical treatment. Re-attend for early morning smear if nucleic acid amplification testing (NAAT) was negative and symptoms do not settle.

- All men assessed for sexually transmitted infections, regardless of symptoms, should be tested for
C. trachomatis from a first-void urine specimen and for Neisseria gonorhoeae if they have urethritis. If a NAAT is positive for gonorrhoea, a culture should be performed before treatment.

- All men who have sex with men should be tested for both $C$. trachomatis and N. gonorrhoeae from any potentially exposed site.

- Recommended syndromic regimen: doxycycline $100 \mathrm{mg}$ twice daily for seven days. Azithromycin $1 \mathrm{~g}$ immediately should not be used routinely because of the increased risk of inducing macrolide antimicrobial resistance with $M$. genitalium.

- If M. genitalium-positive: azithromycin $500 \mathrm{mg}$ immediately, followed by a $250 \mathrm{mg}$ oral dose for four days. A test of cure three to five weeks after treatment in those who tested positive for M. genitalium should be performed.

In case of persistent and/or recurrent non-gonococcal urethritis, testing for $M$. genitalium using a NAAT, preferably with screening for macrolide resistance, should be considered, as well as testing for Trichomonas vaginalis using a NAAT if it is prevalent in the local population. Recurrent non-gonococcal urethritis should only be treated if the patient has definite symptoms of urethritis, or if there are physical signs and microscopic evidence of urethritis on examination.

The guidelines are available here 


\section{References}

1. Horner PJ, Blee K, Falk L, van der Meijden W, Moi H. 2016 European guideline on the management of non-gonococcal urethritis.Int J STD AIDS. 2016;2016(May):4.PMID: 27147267

2. Shahmanesh M, Moi H, Lassau F, Janier M, IUSTI/WHO. 2009 European guideline on the management of male nongonococcal urethritis.Int J STD AIDS. 2009;20(7):458-64. DOI: 10.1258/ijsa.2009.009143 PMID: 19541886

3. Falk L, Fredlund H, Jensen JS. Symptomatic urethritis is more prevalent in men infected with Mycoplasma genitalium than with Chlamydia trachomatis.Sex Transm Infect. 2004;80(4):289-93. DOI: 10.1136/sti.2003.006817 PMID: 15295128

4. Horner P, Blee K, Adams E. Time to manage Mycoplasma genitalium as an STI: but not with azithromycin $1 \mathrm{~g}$ !Curr Opin Infect Dis. 2014;27(1):68-74. DOI: 10.1097/ QC0.0000000000000030 PMID: 24322592

5. Manhart LE, Gillespie CW, Lowens MS, Khosropour CM, Colombara DV, Golden MR, et al. Standard treatment regimens for nongonococcal urethritis have similar but declining cure rates: a randomized controlled trial. Clin Infect Dis. 2013;56(7):934-42. DOI: 10.1093/cid/cis1022 PMID: 23223595

\section{License and copyright}

This is an open-access article distributed under the terms of the Creative Commons Attribution (CC BY 4.0) Licence. You may share and adapt the material, but must give appropriate credit to the source, provide a link to the licence, and indicate if changes were made.

This article is copyright of the authors, 2016. 